\title{
Fallbericht: Aku-Taping bei Achillodynie
}

\author{
Hans Michael Koch
}

\section{Zusammenfassung}

Eine Achillodynie lässt sich häufig erfolgreich durch Aku-Taping, v. a. in Kombination mit Akupunktur therapieren. Gerade Störungen des straffen und kollagenen Bindegewebes, dessen Kollagenfasern bei Sehnen parallelfaserig angeordnet sind, sprechen auf die Reiztherapie durch AkuTaping gut an.

\section{Anamnese}

Die 38-jährige Patientin Frau A. ist seit ihrer Jugend eine passionierte Sportlerin und betreibt leistungsmäßig verschiedene Sportarten. Fußball trainiert sie 2-mal die Woche und hat am Wochenende Ligaspiele. Seit einigen Wochen trainiert sie mit einem neuen Fußballschuh. Seither beklagt die Patientin zunehmende Schmerzen im distalen Anteil der rechten Achillessehne, die zunehmend in die Wade und in die Ferse ausstrahlen. Zuletzt sei Sport deswegen nicht mehr möglich gewesen. Während ihrer Arbeit und zu Hause könne sie sich am besten in Schuhen mit höherem Absatz bewegen.

Lokal zeigt sich eine kirschgroße, druckdolente Schwellung über der distalen rechten Achillessehne und des umgebenden Peritendineums. Bei Bewegung kommt es zu einem palpablen lokalen „Knirschen“. Die Dorsalflexion im oberen Sprunggelenk ist schmerzhaft.

\section{Diagnose}

Bei radiologischem Ausschluss knöcherner Pathologien im Bereich des Kalkaneus besteht die Diagnose einer Achillodynie (ब Kasten) mit lokaler mechanischer Entzündung mit Weichteilschwellung, Schmerz und functio laesa.
Nach Chinesischer Medizin ergibt sich die Diagnose einer lokalen Qi-Stagnation über dem Nieren- und Blasenmeridian mit einer Blutstase und Ansammlung von Nässe-Hitze.

\section{Therapie und Verlauf}

Klassische Therapie: Im akuten Stadium werden in aller Regel Antiphlogistika verordnet und Ruhigstellung durch Gips oder (teil-)immobilisierende Tape-Verfahren (Leukotape $^{\circledR}$ ) angestrebt. Zur Schmerzreduktion werden Lokalanästhetika injiziert.

Im chronischen Stadium wird die Sehne durch eine Absatzerhöhung entlastet. Sportliche Betätigung verbietet sich, eine erneute Belastung erfolgt unter allmählicher Steigerung der Trainingsbelastung. Hilfreich können Iontophorese und Salbenverbände sein. Als Ultima Ratio gilt die chirurgische Intervention mit Exzision des peritendinösen Gewebes.

Frau A. wurde angehalten, zunächst auf Sport weiter zu verzichten und erst bei zunehmender Beschwerdefreiheit das Training langsam wieder zu steigern. Eine normale Alltagsbelastung wurde unter Schmerzberücksichtigung empfohlen.

In den ersten beiden Wochen erfolgte die Behandlung 2-mal wöchentlich, dann über 4 Wochen 1-mal wöchentlich. Zunächst wurde die Patientin nach dem Ketten-Schloss-Prinzip akupunktiert, von proximal nach distal mit dem Verlauf des Blasenmeridians (Bl 40, 57, 58, 60, 62, 64; Abb. 1) und dann von distal nach proximal mit dem Verlauf des Nierenmeridians (Ni 2, 3, 5, 7, 10 rechts; Abb. 2). Alternierend wurden Akupunkturnadeln bis

\section{Achillodynie}

Unter Achillodynie versteht man Schmerzen der Achillessehne, häufig mit entzündlichen Verdickungen der Sehne und eingeschränkter Funktion durch mangelhafte Verschieblichkeiten im Gleitgewebe (Peritendinitis).

Die Mehrzahl der chronischen Beschwerden ist hervorgerufen durch degenerative Veränderungen aufgrund chronischer und inadäquater Überbelastung. Akute Beschwerden treten nach ungewohnten Anstrengungen und durch unzureichendes Schuhwerk (Schaft) auf.

Zudem können systemisch bedingte Stoffwechselanomalien zu durch Fettoder Kristallablagerungen verursachten entzündlichen Reizzuständen führen.

Bei akuter Reizung bestehen erhebliche Beschwerden, die bis zur Gehunfähigkeit führen können. Das obere Sprunggelenk wird ggf. reflektorisch in Spitzfußstellung gehalten. Die mechanische Entzündung des Gleitgewebes um die Achillessehne führt zu Schmerzen v. a. lokal bei Druck und Dehnung (Dorsalextension des Fußes). Ein deutliches Knirschen bei Bewegung sichert die Diagnose.

zum De-Qi und Akupunkturpunktinfiltrationen mit Procain $1 \%$ gesetzt.

Außerdem wurden Gb 34 als Hui-Punkt der Sehnen und Gb30 als Fernpunkt 

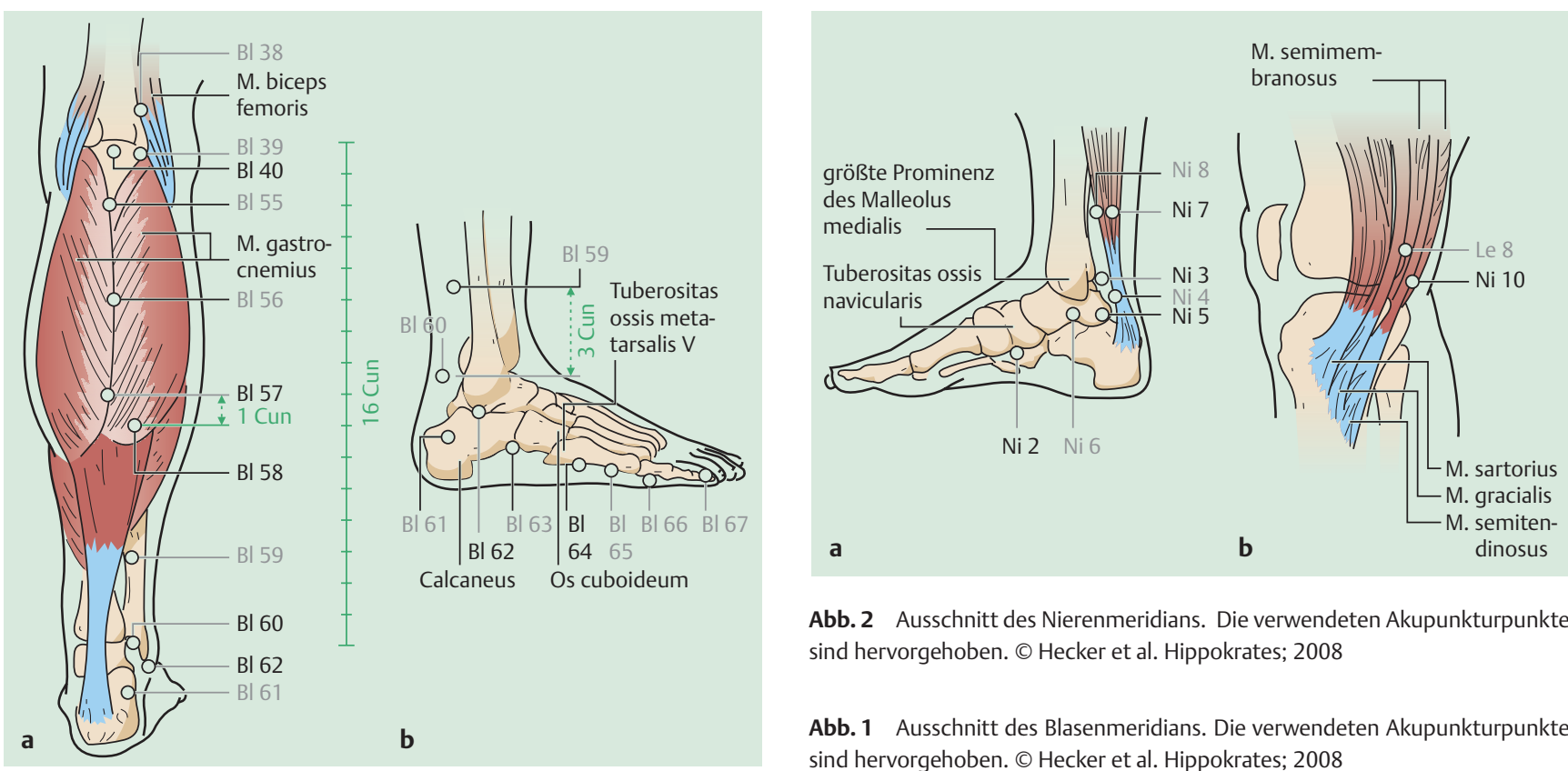

Abb. 2 Ausschnitt des Nierenmeridians. Die verwendeten Akupunkturpunkte sind hervorgehoben. (c) Hecker et al. Hippokrates; 2008

Abb. 1 Ausschnitt des Blasenmeridians. Die verwendeten Akupunkturpunkte sind hervorgehoben. (๑) Hecker et al. Hippokrates; 2008

(Kreuzungspunkt mit dem Blasenmeridian) akupunktiert. Kontralateral wurden als energetischer Ausgleich wenige Akupunkturpunkte auf dem Herz- und Dünndarmmeridian im distalen Unterarm behandelt.

Anschließend wurde das Tape gemäß ( Abb. 3 und 4) fixiert. Die Patientin trug das Tape jeweils über 2-5 Tage. Es kam, je nach Tragedauer, teilweise $\mathrm{zu}$ nicht juckenden leichten Hautrötungen. Frau A. empfand das Tragen des Tapes als sehr angenehm. Nach Abnehmen des Tapes seien die Beschwerden zunächst etwas schlechter geworden. Das ist typisch, wenn die Stimulation durch das reizauslösende Agens wegfällt.

Insgesamt wurde die Patientin 8-mal akupunktiert, die Beschwerden und der Lokalbefund verbesserten sich zunehmend. Drei Wochen nach der letzten Behandlung stellt sich Frau A. dann beschwerdefrei nochmals vor und wünschte ein Tape als prophylaktische Maßnahme im Hinblick auf ein bevorstehendes Turnierwochenende.

\section{Theorie des Tapens}

\section{Anwendung des Tapes}

Grundsätzlich lassen sich Tapes nach dem Medical-Taping-Concept unter verschiedenen Gesichtspunkten platzieren:
- Meridian-Tape,

- Cross-Tape im Bereich von

Akupunktur- und Triggerpunkten,

- Lymph-Tape und

- klassisches Kinesio-Tape.

Beim Aku-Taping werden die Tapes unter Berücksichtigung der betroffenen Meridiane und Akupunkturpunkte geklebt, um nach dem Konzept der Chinesischen Medizin Qi- (und Blut-)Blockaden aufzubrechen und Anhäufungen von Pathogenen aufzulösen. Qi-Blockaden führen zu lokalem Schmerz, Blutstasen und Ansammlungen von Nässe zu Schmerz und palpablen Resistenzen. Diese können u.a. mit einem Hämatom durch Prellung und bei chronischem Verlauf mit Gewebsverquellungen und Pannusgewebe oder Triggerpunkten korrelieren. Bei Störungen des Bewegungsapparats werden die Verläufe der tendinomuskulären Leitbahnen der betroffenen Meridiane berücksichtigt. Die Tapes werden sowohl nach distal und proximal über die schmerzhaften oder bewegungseingeschränkten Strukturen hinaus angelegt. Es sollten jeweils die nächstgelegenen Gelenke miteinbezogen werden, um die Bewegungsumfänge der angrenzenden Gelenke therapeutisch mitzunutzen.

Wie bei der Akupunktur werden Fernpunkte mit einbezogen und jeweils mit

\section{Kleine Historie Aku-Taping}

Das Kinesio-Taping wurde Anfang der 1970er Jahre von dem japanischen Arzt und Chirotherapeuten Kenzo Kase entwickelt. Beim Kinesio-Taping werden Tapes über vorgedehnte Muskel- und Gelenkzonen geklebt.

Hans-Ulrich Hecker und Kay Liebchen entwickelten aus diesen Techniken 2004 das Aku-Taping und beschrieben im Buch Aku-Taping - sanft gegen den Schmerz (Stuttgart: Haug; 2005). AkuTapes werden heute therapeutisch und prophylaktisch auch im Profisport verwendet. Neben der Fußballnationalmannschaft und einigen Bundesligaclubs findet das Aku-Taping publikumswirksam bei der Handballnationalmannschaft Anwendung.

Einzelpunkt-Tapes versehen. So ist die Verwendung eines stark vorgedehnten Cross-Tapes ( 2 ca. $5 \mathrm{~cm}$ lange Einzel-Tapes kreuzförmig geklebt) über Gb 34 bei Beschwerden im Bewegungsapparat hilfreich. 
Tapes „pieken“ nicht und sind so beispielsweise auch bei Kindern nützlich, die eine Nadelakupunktur ablehnen.

Aku-Tapes bestehen aus einer luft- und wasserdurchlässigen Baumwolltextur und sind hautseitig mit einem Acrylkleber versehen. Dieser ist thermoaktiv und entfaltet die volle Klebwirkung erst nach einiger Zeit des Tragens. Sie werden in verschiedenen Farben und Breiten von verschiedenen Herstellern angeboten.

Tapes haben eine Längselastizität mit einer Dehnbarkeit von ca. 50\%, aber keine Querelastizität. Sie werden unter maximaler Dehnung der betroffenen anatomischen Strukturen und unter leichter Dehnung des Tapes selber fixiert. Die Enden werden immer rund geschnitten, um vorzeitiges Ablösen bei vermehrter mechanischer Belastung über die sonst bestehenden Ecken zu vermeiden.

Die Tapes werden, wenn sie keine lokalallergischen Erscheinungen provozieren, ein bis mehrere Tage belassen. Dann werden sie vorsichtig, am besten nass oder ölig, von der Haut gelöst.

\section{Wirkweise des Tapes}

Das Aku-Tape immobilisiert nicht und sitzt wie eine „zweite Haut“. Durch die Dehnung von Haut und unterliegenden Schichten und der Dehnung des Tapes bei der Fixierung kommt es bei Kontraktion der unterliegenden Muskulatur zu einer Anhebung von Haut und unterliegenden Bindegewebsanteilen. Diese manifestiert sich als Faltenbildung des Tapes und der daran festklebenden Haut. Bei Dehnung der unterliegenden Muskulatur hingegen wird ein Druck in die Tiefe aufgebaut. Diese unterschiedlichen Kräfte bewirken unter der Tragedauer eine unablässige Steigerung des lokalen Stoffwechsels auf ca. $150 \%$. Die Patienten berichten unter Tape häufig von einem vermehrten Wärmeempfinden. Durch die „Isolation“ der unterliegenden Haut kommt es zu einer gesteigerten Temperatur, die zudem zu einer kompensatorischen Weitstellung der lokalen Blutgefäße führt.

Das Tape führt zudem zu einer Vermehrung des lokalen Lymphflusses und wirkt durch Druckauf- und -abbau ähnlich wie die Muskel-Arterien-Pumpe positiv auf den Abtransport der Lymphe und dadurch

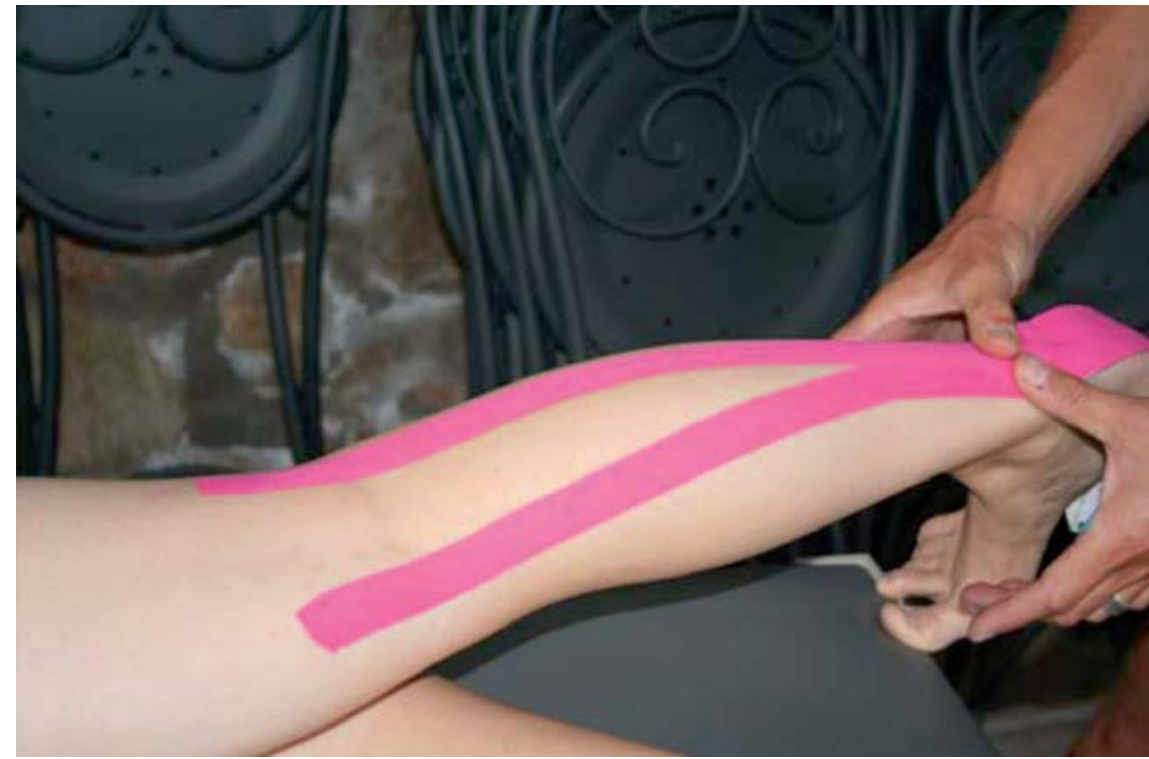

Abb. 3 Fixieren des Y-Tapes unter Dehnung, Blasen- und Nierenmeridian. ㄷ H. M. Koch

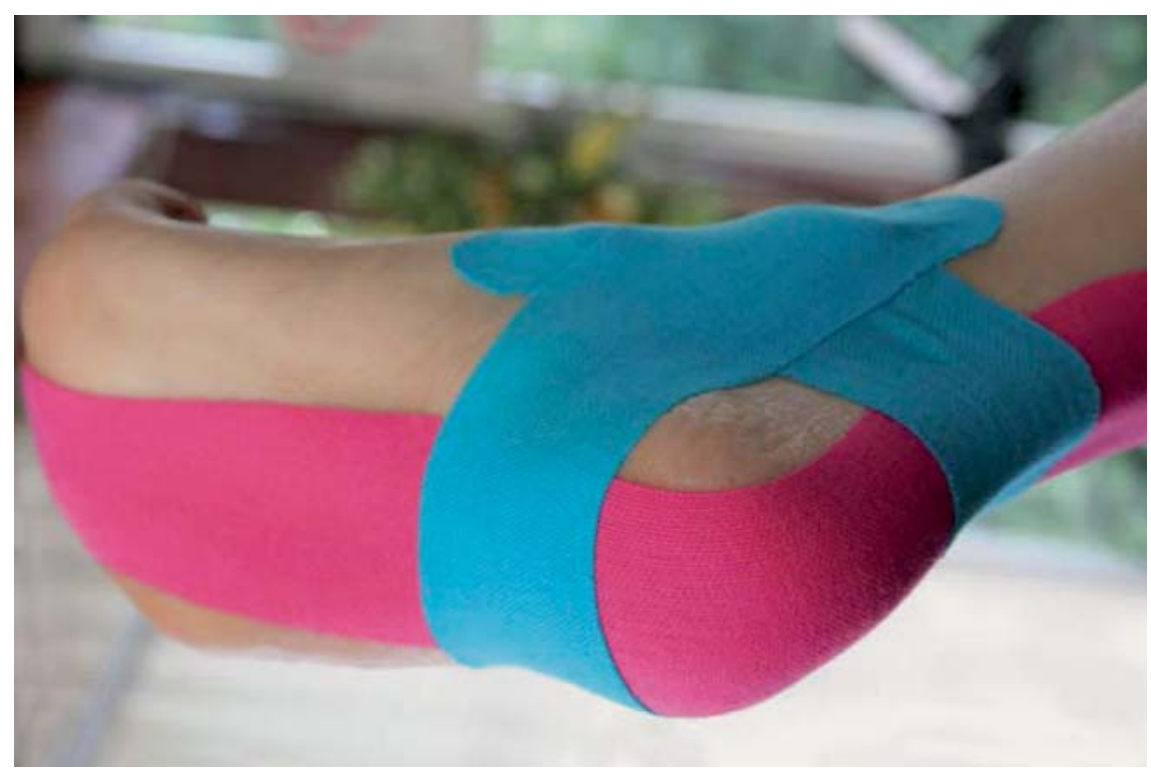

Abb. 4 Quer-Tapes im Bereich über der Achillodynie und den lokalen Akupunkturpunkten von Blasen- und Nierenmeridian. (c) H. M. Koch

drainierend. Dem Verkleben der Schichten und dem Umbau des Gerüsteiweißes zu dickeren und derberen Fasern bei Inaktivität wird so entgegengewirkt. Gewebsverdickungen, Lymphödeme und Gelenksergüsse werden innerhalb kurzer Zeit messbar kleiner.

Unzählige von Exterozeptoren der Haut und Propriozeptoren im Bindegewebe werden unter dem Tape bei Bewegung ständigem und vermehrtem Reiz ausge- setzt. Durch die Stimulation dieser oberflächlichen Rezeptoren und von afferenten Nervenbahnen kommt es zur Unterdrückung der Schmerzwahrnehmung und zu einer verbesserten reflektorisch gesteuerten Koordination der Muskulatur. Diesen besseren Halt „erzeugen“ die Patienten allerdings selbst, Muskulatur und Funktion werden dadurch trainiert. So kommt es im Gegensatz zu Gipsprothesen und immobilisierenden starren Tapes nicht durch 
erzwungene Ruhe zu Hypotrophie und Unterfunktion, die dann wieder mühsam und kostenintensiv behoben werden müssen.

Anders als beim Kinesio-Taping sind beim Aku-Taping auch interne und psychische Störungen durch das „Kleben“ zugänglich. Wahre Wunder beispielsweise lassen sich bei abdominellen Distensionen durch intraintestinale Gasansammlungen durch das sog. „Schräge- und Gerade-Bauchmuskel-Tape“ erzielen.

\section{Verwendete Literatur}

[1] Niethard FU, Pfeil ]. Orthopädie. Duale Reihe. 5. Aufl. Stuttgart: Thieme; 2005

[2] Debrunner AM. Orthopädie, Orthopädische Chirurgie. Bern: Hans Huber; 1994

[3] Hecker HU, Liebchen K. Aku-Taping - sanft gegen den Schmerz. Stuttgart: Haug; 2005

[4] Groth KJ, Gericke R-E. Kleb den Schmerz einfach weg. München: Herbig Verlagsbuchhandlung; 2005

[5] Schünke M, Schulte E, Schuhmacher U. Prometheus Allgemeine Anatomie und Bewegungssystem. Stuttgart: Thieme; 2007

[6] Wittlinger $\mathbf{H}$, Wittlinger $\mathbf{C}$. Lehrbuch der manuellen Lymphdrainage nach Dr. Vodder. Heidelberg: Haug; 1996

\section{Weiterführende Informationen}

\section{TCM-Ausbildung}

koch.kurse über das TCM-Ausbildungszentrum der Johanniter JohanniterKrankenhaus Bramsche, Hasestraße 16-18, 49565 Bramsche Tel. 05461/805127

www.aku-taping.de

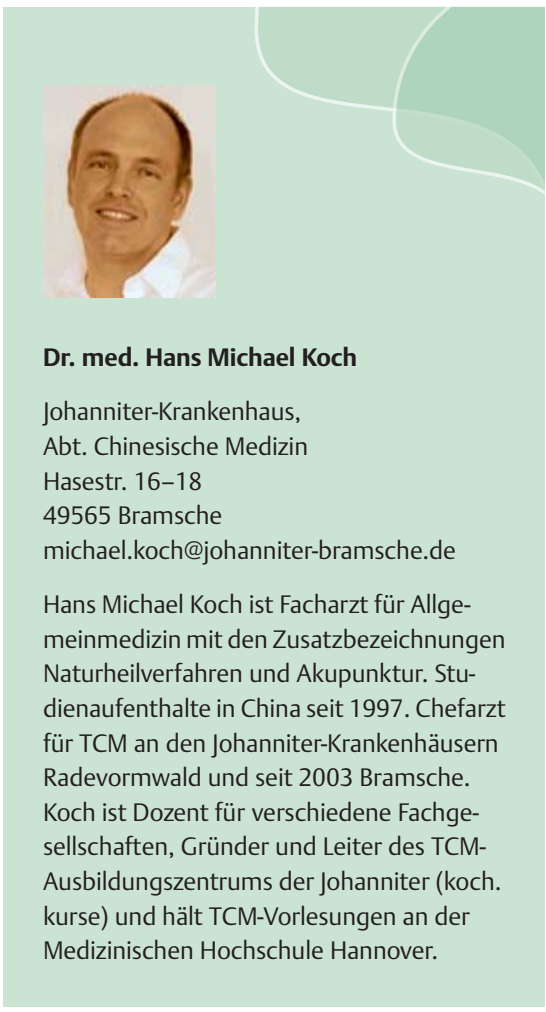

\section{Effektive Hilfe für Ihre Schmerzpatienten}
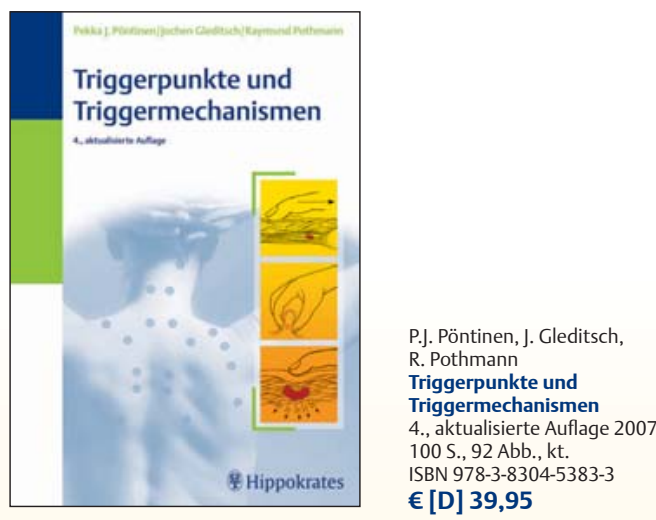

Wesentliches der Triggerpunktlehre mit Therapieansätzen aus der Akupunktur: von den Triggermechanismen über die Lokalisierung der Triggerpunkte, die wichtigsten Triggermuskeln, Triggerpunktsyndrome und Triggerpunkt-(Reflex-)Systeme bis hin zu unterschiedlichen Techniken der Triggerbehandlung.

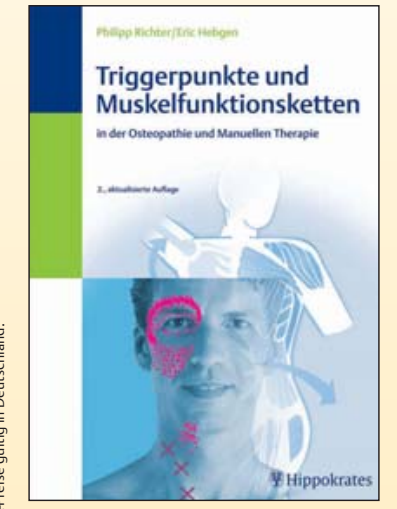

\section{Bestseller!}

Lernen Sie die verschiedenen Konzepte von Muskelfunktionsketten kennen und erfahren Sie, welchen Bestand die einzelnen Konzepte in der täglichen osteopathischen und manualtherapeutischen Arbeit haben.

Die exakte Lokalisation der Triggerpunkte und ihrer zugehörigen Schmerzareale wird Ihnen durch anatomische Übersichten und zahlreiche Fotos erleichtert.

MVS Medizinverlage Stuttgart $\mathrm{GmbH} \&$ Co. KG

Oswald-Hesse-Straße 50

70469 Stuttgart

Tel. (0711) 8931-900

Fax (0711) 8931-901 kundenservice@thieme.de www.medizinverlage.de 